\title{
Trans-ethnic study design approaches for fine-mapping
}

\author{
Jennifer L Asimit ${ }^{1}$, Konstantinos Hatzikotoulas ${ }^{1}$, Mark McCarthy ${ }^{2}$, Andrew P Morris ${ }^{2,3}$ and \\ Eleftheria Zeggini ${ }^{\star}, 1$
}

Studies that traverse ancestrally diverse populations may increase power to detect novel loci and improve fine-mapping resolution of causal variants by leveraging linkage disequilibrium differences between ethnic groups. The inclusion of African ancestry samples may yield further improvements because of low linkage disequilibrium and high genetic heterogeneity. We investigate the fine-mapping resolution of trans-ethnic fixed-effects meta-analysis for five type II diabetes loci, under various settings of ancestral composition (European, East Asian, African), allelic heterogeneity, and causal variant minor allele frequency. In particular, three settings of ancestral composition were compared: (1) single ancestry (European), (2) moderate ancestral diversity (European and East Asian), and (3) high ancestral diversity (European, East Asian, and African). Our simulations suggest that the European/Asian and European ancestry-only meta-analyses consistently attain similar fine-mapping resolution. The inclusion of African ancestry samples in the meta-analysis leads to a marked improvement in fine-mapping resolution. European Journal of Human Genetics (2016) 24, 1330-1336; doi:10.1038/ejhg.2016.1; published online 3 February 2016

\section{INTRODUCTION}

Numerous genome-wide association studies (GWASs) have been carried out, resulting in the identification of many susceptibility loci for a wide range of complex traits. ${ }^{1}$ The detection of additional loci has resulted from GWAS meta-analyses (primarily in populations of European descent) and has been aided by imputation that allows the prediction of genotypes not typed on GWAS chips, but present in a higher density reference. Nonetheless, the joint effects of the loci identified to date have only accounted for a small proportion of the heritability of complex traits. Because of linkage disequilibrium (LD), many variants within identified loci have indistinguishable signals. This LD is beneficial to GWAS, as it increases the power to detect new associations, when the causal variant is not directly typed. However, the caveat to this is that it limits the potential of fine-mapping efforts to refine the location of causal variants.

GWAS data from non-European populations are increasing in availability, and this provides the opportunity to meta-analyse GWAS across ancestrally diverse populations. Trans-ethnic meta-analysis may lead to an increase in power to detect novel loci and may improve fine-mapping resolution of causal variants by leveraging differences in the structure of LD between diverse populations. ${ }^{2,3}$ The inclusion of African ancestry samples may yield substantial improvements in localisation of causal variants because of low LD and high genetic heterogeneity. ${ }^{4}$

Several trans-ethnic analyses have shown empirical improvements in fine-mapping resolution. Examples include a refinement of signals at several type II diabetes loci in an analysis involving samples of European, East Asian, South Asian, and Mexican and MexicanAmerican ancestry, ${ }^{5}$ as well as in previously identified adiposity loci in a trans-ethnic meta-analysis of samples from European and African ancestries. ${ }^{6}$ Similarly, a refinement of signals was obtained at several lipid trait loci in a trans-ethnic fine-mapping study involving African-
American, East Asian, and European ancestries. ${ }^{7}$ However, a comparison of study designs, where the ancestral composition of the samples varies in diversity whereas the same number of samples (and cost) is constant, has not yet been performed. Approaches to meta-analysis include a fixed- or random-effects model, as implemented in GWAMA (genome-wide association meta-analysis ${ }^{8}$ ) or MANTRA (Meta-ANalysis of Trans-ethnic Association studies), that allows for heterogeneity in allelic effects between distantly related populations. ${ }^{9}$ We focussed on fixed-effects meta-analysis, as trans-ethnic studies of complex traits demonstrated relatively little evidence of heterogeneity in allelic effects at common variant loci. Moreover, MANTRA and fixed-effects GWAMA have been shown to have similar power when the variant has a non-null effect in all of the populations. ${ }^{10}$ Therefore, our objective is to examine different study designs with respect to ancestral composition, rather than statistical approaches to metaanalysis. There may be power and fine-mapping advantages in increasing the ancestral diversity among the samples while retaining the same number of samples and cost.

We carried out an extensive simulation study to examine different study designs with respect to ancestral composition (European, East Asian, and African) to assess any power and fine-mapping advantages due to increasing ancestral diversity among samples while retaining the same number of samples and cost. The aim is to determine whether trans-ethnic meta-analyses offer fine-mapping resolution advantages over single ancestry meta-analyses, and whether or not any gains are due to the inclusion of African ancestry samples.

\section{MATERIALS AND METHODS}

We focus on five established type II diabetes (T2D) loci (IGF2BP2, CDKN2A/B, KCNQ1, FTO, CDKAL1). These loci were explicitly chosen as they have association signals present in multiple ethnic groups, ${ }^{11}$ and several of these loci have shown large differences in LD between ethnic groups (eg, CDKAL1 and

${ }^{1}$ Wellcome Trust Sanger Institute, Cambridge, UK; ${ }^{2}$ Wellcome Trust Centre for Human Genetics, University of Oxford, Oxford, UK; ${ }^{3}$ Department of Biostatistics, University of Liverpool, Liverpool, UK

${ }^{*}$ Correspondence: Professor E Zeggini, Wellcome Trust Sanger Institute, The Morgan Building, Wellcome Trust Genome Campus, Hinxton, Cambridge CB10 1HH, UK. Tel: +44 1223 496868; Fax: +44 1223 496826; E-mail: eleftheria@sanger.ac.uk

Received 2 April 2015; revised 22 October 2015; accepted 22 December 2015; published online 3 February 2016 
KCNQ1). Such loci characteristics are likely to favour success for trans-ethnic meta-analyses.

In this simulation study, trans-ethnic meta-analysis was carried out by a frequentist fixed-effects meta-analysis that was implemented in GWAMA $^{8}$ as a proof of principle in comparing fine-mapping of studies having varying degrees of ancestral diversity. In addition, for completeness, we also employed GWAMA using random effects in a selection of settings. Fine-mapping assessment was examined when the variant had a non-null effect in all of the populations and under various levels of ancestral diversity: European-only samples; moderate ancestral diversity (European and Asian samples); and high ancestral diversity (European, East Asian, and African samples).

\section{Simulation settings}

Sets of six cohorts were meta-analysed across five loci under various allelic heterogeneity models and ancestry compositions of the contributing cohorts. Data were simulated using Hapgen $2^{12}$ from 6 populations based on the 1000 Genomes June 2011 haplotypes: ${ }^{13}$ CEU, TSI (European reference panels (RPs)), CHB, JPT (East Asian RPs), LWK, and YRI (African RPs). Each cohort was composed of 1000 cases/1000 controls, and 1000 replications were used for each setting. These simulations represent directly typed data, and variants are referred to as perfect. They were then thinned down to GWAS density based on the SNPs in the Illumina 660-Quad array, and subsequently imputed via IMPUTE2 ${ }^{14}$ using the same cross-population 1000 Genomes reference panel, and effective population size $\mathrm{Ne}=20000$. A $500 \mathrm{~kb}$ up- and down-stream buffer was included in the imputation, and variants with SNPTEST proper information score below 0.4 were filtered out. Both perfect (all variants are directly typed) and imputed data were analysed. Analysis of the perfect data illustrates an optimal scenario and provides the maximum possible power and refined fine-mapping resolution that may be attained, whereas the imputed data represent a more realistic setting.

There were three general ancestry combinations considered for metaanalysis:

1. Single ancestry (European): $6 \mathrm{CEU}$ samples;

2. Moderate ancestral diversity (European and Asian): 3 CEU+TSI samples, $3 \mathrm{CHB}+\mathrm{JPT}$ samples; and

3. High ancestral diversity (European, Asian, and African): 1 sample from each of CEU, TSI, CHB, JPT, LWK, and YRI.

Within each locus, causal variants were selected within $\pm 0.5 \%$ of one of three minor allele frequencies (MAFs): 5,10 , or $20 \%$, with respective relative risks of 1.4, 1.3, and 1.2. As the MAF varies between the populations, when there was a shared causal variant $c_{1}$, it was selected to satisfy the MAF requirements in the $\mathrm{CEU}$ population frequency and to be nonmonomorphic in the other populations. A single causal variant $c_{1}$ was considered for each of the single ancestry and moderate and high ancestral diversity scenarios.

\section{Meta-analysis and fine-mapping}

Within each study sample, single SNP association tests were carried out with an additive model in a 'missing data likelihood' framework, as implemented in SNPTEST v2. ${ }^{15}$ As put into practice in GWAMA, ${ }^{8}$ the frequentist fixed-effects meta-analysis of the single SNP summary statistics assumes that the allelic effect at SNP $j$ is the same across all samples. This meta-analysis approach is highly computationally efficient, but has the limitation that it assumes a homogeneous effect size among all of the samples.

A measure of fine-mapping resolution was based on the construction of $95 \%$ credible sets using Bayesian theory, such that the sets were 95\% likely to encompass the causal SNP. In order to form the credible sets, the association summary statistics ( $\hat{\beta}$ and its variance, $V$ ) from each SNP were first converted to an approximate Bayes' factor $(\mathrm{ABF})$ :

$$
\mathrm{ABF}=\sqrt{\frac{\mathrm{V}}{V+W}} \operatorname{Exp}\left\{\frac{\mathrm{W}}{V+W} \times \frac{\mathrm{Z}^{2}}{2}\right\}
$$

where $Z=\frac{\hat{\beta}}{\sqrt{V}}, \hat{\beta} \sim N(\beta, V), \beta \sim N(0, W)$ and $N\left(\mu, \sigma^{2}\right)$ denotes that the random variable follows a Normal distribution with mean $\mu$ and variance $\sigma^{2}{ }^{16}$ For a case-control study and an additive model, a widely used default value is
$W=0.04 .^{15}$ Evidence against the null hypothesis increases with the ABF value, and posterior probabilities were calculated from the ratio of the individual ABF value to the sum of the ABF values for all SNPs within the region. These posterior probabilities were then ranked in decreasing order and SNPs were consecutively collected to form a $95 \%$ credible set until the total of the posterior probabilities for the SNPs in the set first exceeded $0.95 .{ }^{17}$

We only constructed $95 \%$ credible sets when the lead SNP had a $P$-value below a prespecified threshold in order to avoid the construction of sets consisting only of SNPs that are unlikely to be disease associated. The construction of credible sets based on lead SNPs with weak signals requires a larger number of SNPs to meet the criterion of a certain total posterior probability, as the individual SNP posterior probabilities tend to be small in this case. For computational efficiency, we focussed on a frequentist threshold for the decision of whether or not to construct a credible set. This avoided an additional computational step of $\mathrm{ABF}$ calculations that would not be required if it was inappropriate to construct a credible set.

In assessing any effect of ancestral diversity on fine-mapping resolution, the probability of the causal variant being a member of the $95 \%$ credible set was assessed among simulation scenarios in which all samples shared a causal variant. For each region, the median number of SNPs within credible sets that contained the true causal variant was considered in evaluating the refinement for localising the causal variant within the locus; a smaller number is indicative of higher fine-mapping resolution. As a means of combining the measures over the regions, we refer to the average of the region-specific medians as a summary statistic.

Effects of allelic heterogeneity were investigated by introducing a different causal variant $c_{2}$ for the Asian samples, such that it was of similar MAF, but in low LD $\left(r^{2}<0.2\right)$ with the European causal variant. In particular, for the moderate ancestral diversity scenario, we compared the setting of no heterogeneity, where $c_{1}$ is the causal variant for both European and East Asian samples, with the allelic heterogeneity setting of causal variant $c_{1}$ for European samples and causal variant $c_{2}$ for East Asian samples.

To reflect locus heterogeneity, in the high ancestral diversity set-up we assumed null associations at both $c_{1}$ and $c_{2}$ for the African samples; we refer to such cohorts as null. In these heterogeneity scenarios we examined the impact on power to detect the variant that is causal in the European samples. We compared the settings of no heterogeneity (causal variant $c_{1}$ in all samples), allelic and locus heterogeneity (causal variants $c_{1}$ in European samples and $c_{2}$ for East Asian samples; null for African samples), and locus heterogeneity (causal variant $c_{1}$ in European samples; null for East Asian and African samples). For both types of heterogeneity, comparisons are also made with the power to detect a causal variant in the single ancestry (European) composition to assess the power loss of trans-ethnic meta-analyses in the presence of heterogeneity.

\section{RESULTS}

In the decision for construction of $95 \%$ credible sets, a stringent lead SNP $P$-value threshold of $5 \times 10^{-8}$ was employed. The probability that the $95 \%$ credible set contained the true causal variant was high across all five loci and there was no clear collective pattern between probabilities and degree of ancestral diversity. Upon collapsing the individual region results into averages over the regions, any differences were smoothed over and there were negligible differences between the probabilities for the various diversity settings at a given causal variant MAF (Table 1). For the high diversity scenario with perfect data, a random-effects meta-analysis was also implemented using GWAMA. The summary measure over the five regions of the probability that the 95\% credible set contains the causal variant drops slightly with random effects, compared with fixed effects, and the standard error of these estimates are slightly higher for random effects. The respective summary probabilities for MAFs 5,10 , and $20 \%$ are $0.911,0.900$, and 0.912 (with standard errors 0.00437, 0.00453, and 0.00489) for random effects, whereas the coinciding probabilities for fixed effects 
Table 1 Summary measure (and SE) over the five regions of the probability that the causal variant is in the $95 \%$ credible set

\begin{tabular}{lcccc}
\hline Ancestry combination & Data type & MAF 5\% & MAF 10\% & MAF 20\% \\
\hline Single (Eu) & Perfect & $0.944(0.00367)$ & $0.894(0.00447)$ & $0.929(0.00426)$ \\
& Imputed & $0.864(0.00768)$ & $0.815(0.00650)$ & $0.892(0.00562)$ \\
Moderate (Eu/EA) & Perfect & $0.922(0.00558)$ & $0.846(0.00442)$ & $0.00567)$ \\
High (Eu/Ea/Af) & Imputed & $0.894(0.00644)$ & $0.910(0.00421)$ & $0.90410)$ \\
& Perfect & $0.930(0.00395)$ & $0.845(0.00559)$ & $0.00490)$ \\
Single (Af) & Imputed & $0.892(0.00517)$ & $0.929(0.00404)$ & $0.928(0.00440)$ \\
\hline
\end{tabular}

Perfect and imputed data results are given in the upper and lower portion of each cell, respectively. Credible sets were constructed only when the lead SNP had $P$-value below $5 \mathrm{E}-08$.

are $0.930,0.910$, and 0.928 (with standard errors 0.00395, 0.00421, and 0.00440 ).

Next, we examined the median number of SNPs in the credible sets for each region. Summary measures over the regions are provided in Table 2, and individual region results are available in Supplementary Table S1. Boxplots displaying the distribution of the number of SNPs in $95 \%$ credible sets are given for summary measures and individual loci in Figures 1 and 2 for perfect and imputed data, respectively. That is, each summary measure boxplot gives the distribution of the median number of SNPs in the $95 \%$ credible sets constructed for each locus, whereas the individual loci boxplots display the distributions for the number of SNPs in the $95 \%$ credible sets. The summary statistics suggested that the high ancestral diversity meta-analysis had the smallest median number of SNPs in 95\% credible sets, regardless of causal variant MAF and whether or not the data were imputed; at MAF $10 \%$, for increasing levels of diversity, the perfect data summary medians were 2,3 , and 1 , whereas for imputed data they were 3.6, 3 , and 1.6 (Table 2). In most of the individual region analyses, the highly diverse ancestral composition meta-analysis achieved the smallest median or the same median as the moderate ancestral diversity meta-analysis (Supplementary Table S1). In the random-effects metaanalyses of the high diversity setting for perfect data, the summary measures over the five loci for the median number of SNPs in the 95\% credible sets were 1.6, 1.0, and 2.8 at MAFs 5, 10, and 20\%, respectively; the credible sets constructed from the random-effects meta-analyses are similar to those for fixed effects. The locus-level results are not presented for this random-effects (RE) analysis, as they are identical to the fixed-effects (FE) analysis, except for smaller medians at MAF 20\% for CDKAL1 (RE 5 SNPs; FE 6 SNPs) and at MAF 5\% for FTO (RE 4 SNPs; FE 5 SNPs).

Within each region we used the Kruskal-Wallis rank sum test to assess the evidence for the null hypothesis that the median was the same in each ancestral setting group against the alternative that at least one group had a different median. If there was evidence of a difference, Dunn's test was then applied to test each of the pairwise comparisons for the null hypothesis that, with probability 0.5, a random SNP count from the first group is larger than one from the second group. This may be interpreted as a test for one group having a larger median than the other.

The Kruskal-Wallis test, applied to perfect data, resulted in strong evidence $\left(P\right.$-value $\left.<10^{-5}\right)$ that at least one ancestral setting had a median SNP count different from the others, with the exception of one region and MAF setting. Similarly high levels of significance $\left(P\right.$-value $\left.<10^{-5}\right)$ were attained for all imputed data settings.

In both perfect and imputed data, for each region with a causal variant having MAF $10 \%$ or $20 \%$, Dunn's test gave strong evidence $\left(P\right.$-value $\left.<10^{-5}\right)$ of the high ancestral diversity group having a lower
Table 2 Summary measure of the medians over the five regions for the regional median number of SNPs in the $95 \%$ credible sets

\begin{tabular}{lcccc}
\hline Ancestry combination & Data type & MAF 5\% & MAF 10\% & MAF 20\% \\
\hline Single (Eu) & Perfect & 4.6 & 2.0 & 7.8 \\
& Imputed & 8.2 & 3.6 & 9.0 \\
Moderate (Eu/EA) & Perfect & 3.8 & 3.0 & 10.8 \\
& Imputed & 4.2 & 3.0 & 11.6 \\
High (Eu/Ea/Af) & Perfect & 1.8 & 1.0 & 3.0 \\
& Imputed & 2.4 & 1.6 & 4.6 \\
Single (Af) & Perfect & 1.0 & 1.0 & 1.6
\end{tabular}

Perfect and imputed data results are given in the upper and lower portion of each cell, respectively. Credible sets were constructed only when the lead SNP had $P$-value below $5 \mathrm{E}-08$.

median than the single ancestry group. This was also true at the lower MAF for each region, except for only moderate evidence at $C D K N 2 A / B$ (perfect and imputed data $P$-values 0.0302 and 0.0973). There was not a clear trend between the European and European/ Asian meta-analyses, as either group was found to have strong support $\left(P\right.$-value $\left.<10^{-5}\right)$ for a larger median or no evidence $(P$-value $>0.1)$ for a difference in medians for various MAFs and regions.

As each gene may differ in LD structure, we paired the medians of each ancestral diversity comparison according to gene, and then tested whether the region medians of lower ancestral diversity settings had a greater average than those of higher ancestral diversity via a paired $t$-test. Detailed results are given in Supplementary Table S2. For perfect data, over all MAF settings, there was moderate evidence (considering the small sample size of 5 regions) that the regional median numbers of SNPs in credible sets were on average larger in European-only meta-analyses than in those that include African and East Asian ancestry samples ( $P$-values $0.071,0.045$, and 0.035 for MAFs 5, 10, and 20\%, respectively). Imputed data had similar levels of evidence ( $P$-values 0.023 and 0.047 for MAFs 5 and 20\%, respectively) and considering 0.05 Bonferroni-corrected threshold of $\alpha=0.011$ for the three pair-wise comparisons, this holds with strong evidence for MAF $10 \%$ ( $P$-value 5.5E-03). The results also suggested that the European/Asian trans-ethnic meta-analysis did not have a greater fine-mapping refinement than the European-only meta-analysis, except for moderate evidence in the low MAF setting of imputed data ( $P$-value 0.032$)$. There was moderate evidence for a higher median in European/Asian compared with European/Asian/African at MAF 5\% ( $P$-values 0.051 and 0.069 for perfect and imputed data, respectively).

As we found a clear improvement in the trans-ethnic meta-analyses composed of European, East Asian, and African populations, but not in those without African samples, we also investigated fine-mapping in 

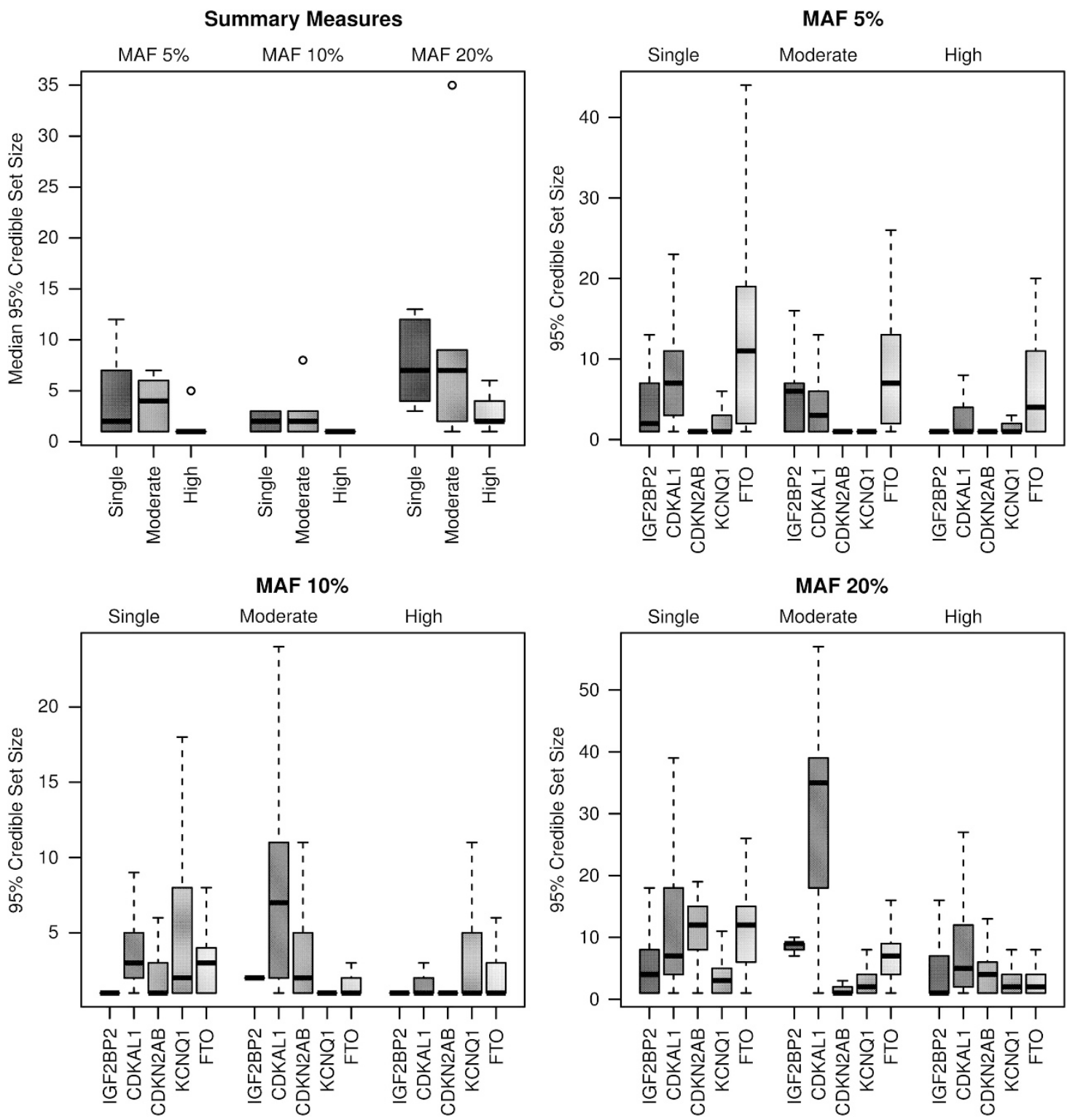

Figure 1 Boxplots, for perfect data, of the number of SNPs in the 95\% credible sets for summary measures of the five loci and for individual loci at MAFs 5 , 10 , and $20 \%$ and ancestral diversities single, moderate, and high.

African-only meta-analyses of perfect data. Based on the same simulation setting of 1000 cases/1000 controls for each sample and the same causal variants (based on European populations), where three samples are taken from each of the LWK and YRI populations, we found that the proportion of $95 \%$ credible sets that contain the causal variant is similarly high for the various ancestral diversity settings. However, the standard errors of these probabilities are slightly higher for the African-only samples, as there are fewer replications in which the lead SNP passes the $P$-value threshold of $5 \times 10^{-8}$ (Table 1). In addition, the median tends to be lowest in the African-only metaanalyses (see Table 2 for summaries and Supplementary Table S1 for individual loci).

Fine-mapping resolution is most refined for African-only metaanalyses, suggesting that features of the African populations such as lower LD rather than ethnic diversity are the underlying source of improvement in the high diversity settings. The allele spectrum between the populations was also examined; for the various European causal variant MAF settings, the median MAFs for the East Asian and African samples are provided in Supplementary Table S3. The median MAF in the African samples tends to be larger than that of the European samples, whereas the median MAF in the East Asian samples tends to be similar or larger in comparison with European samples. However, there is no clear pattern related to MAF and finemapping improvement, indicating that this improvement is more related to LD.

We also examined the effects of ancestral diversity on power to detect an association with a variant that was causal in all samples. At significance level 0.05, regardless of the level of ancestral diversity among the samples, a power close to or identically $100 \%$ was attained for both perfect and imputed data, respectively. Upon repeating the meta-analysis with random effects for IGF2BP2 in the high ancestral diversity setting and for the three MAF settings, identical power results of 1.0 were attained for perfect data. 

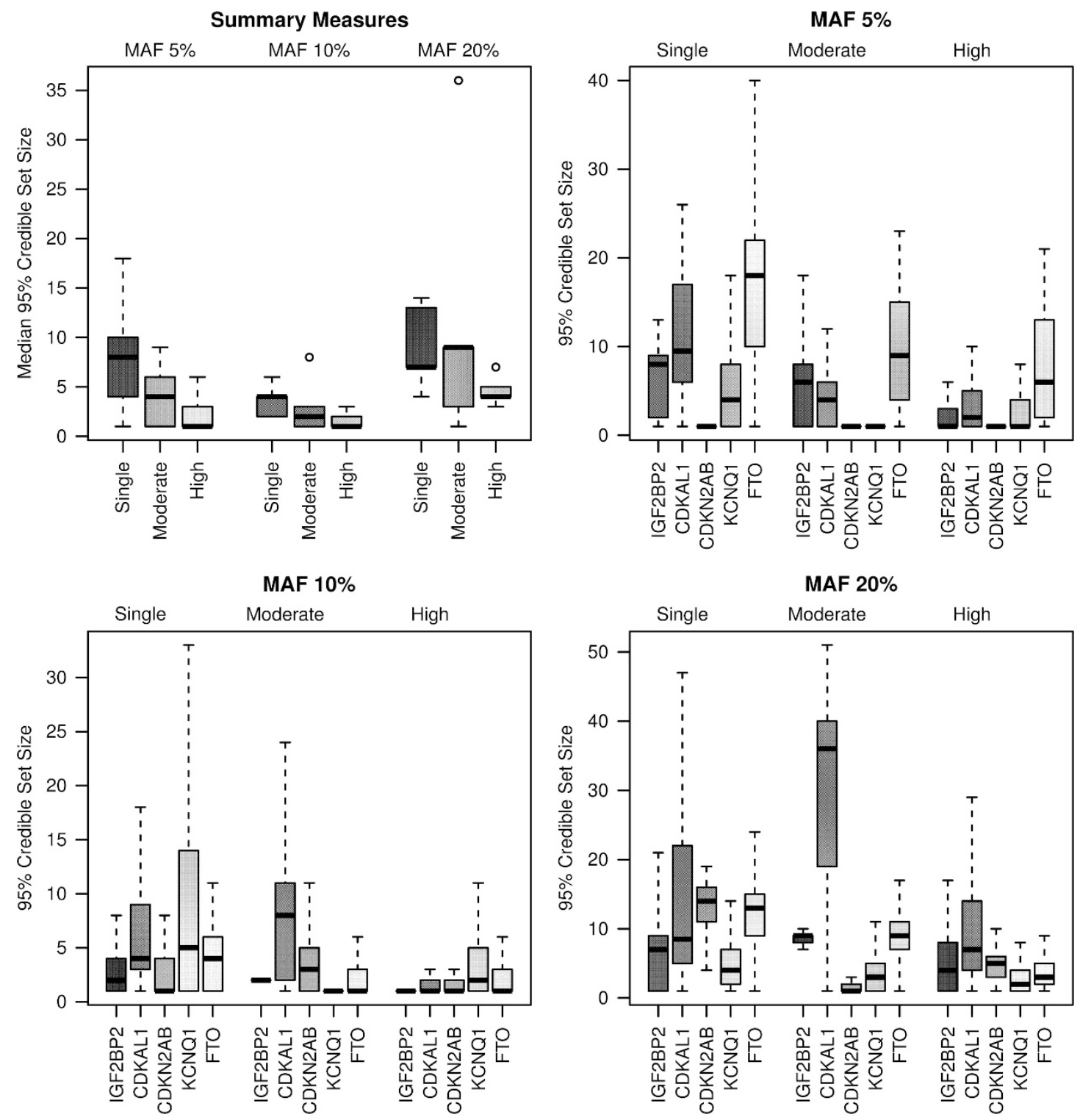

Figure 2 Boxplots, for imputed data, of the number of SNPs in the 95\% credible sets for summary measures of the five loci and for individual loci at MAFs 5,10 , and $20 \%$ and ancestral diversities single, moderate, and high.

For imputed data, the causal variant may either be a tag SNP, so that it is directly typed, or it may be imputed. Among the replications for imputed data, upon examination of the power only at the imputed causal variants, there are not substantial differences compared with the power at directly typed variants. For instance, when the causal variant is the same across all ancestries and diversity settings, the power remains close to 1.0 at imputed-only causal variants, except that at MAF 5\% the power is slightly lower for single ancestry in FTO $(4 \%$ lower) and in $C D K N 2 A B$ ( $8 \%$ lower). In the presence of locus or allelic heterogeneity, there are also very few differences in power between imputed and directly typed causal variants; at MAF 5\%, there is occasionally a power reduction near $10 \%$ when causal variants are imputed rather than directly typed. As the differences are not substantial, this subset of results from the imputed data is not provided.

The effect of allelic heterogeneity on the power to detect a causal variant was assessed by comparing the moderate ancestral diversity scenario in which both Europeans and Asians shared the same causal variant, against the setting in which they had different causal variants in low LD with one another. In the moderate diversity setting, power losses from the presence of allelic heterogeneity in half of the samples ranged from $10 \%$ in perfect data up to $23 \%$ in imputed data, irrespective of MAF (Table 3); these same losses are observed between single ancestry and allelic heterogeneity in the moderate diversity setting when the data are perfect and at MAFs 10 and 20\% in imputed data. Specifically, in individual regions, perfect data had power losses from allelic heterogeneity between 3.8 and $26.2 \%$, with median $12.3 \%$. For imputed data, power losses from moderate diversity with heterogeneity over that without heterogeneity (or single ancestry) are between $5.7 \%(5.6 \%)$ and $33.2 \%(33.2 \%)$ with median $16.4 \%$ (16.2\%). At MAF 5\%, in imputed data, the power for the single ancestry setting is on average $2.6 \%$ lower than that of the setting in which there is no heterogeneity in the moderate diversity setting. Upon repeating the meta-analysis with random effects for IGF2BP2 in the presence of allelic heterogeneity and for perfect data, there was a larger power reduction; fixed-effects meta-analyses yielded powers of 
Table 3 Over the five regions, the mean power to detect the variant that is causal in the European samples $c_{1}$ (at significance level 0.05 ) in the absence/presence of allelic heterogeneity; positive detection occurs when the $P$-value for the single variant test at $c_{1}$ is below 0.05

\begin{tabular}{|c|c|c|c|c|}
\hline Sample composition and causal & Data & $M A F$ & $M A F$ & $M A F$ \\
\hline variants & type & $5 \%$ & $10 \%$ & $20 \%$ \\
\hline \multirow[t]{2}{*}{ Eu-c $c_{1}$ (single ancestry) } & Perfect & 1.000 & 1.000 & 1.000 \\
\hline & Imputed & 0.970 & 0.999 & 0.999 \\
\hline \multirow[t]{2}{*}{$\mathrm{Eu} / \mathrm{EA}-\mathrm{c}_{1}$ (no heterogeneity) } & Perfect & 1.000 & 1.000 & 1.000 \\
\hline & Imputed & 0.996 & 1.000 & 0.999 \\
\hline \multirow[t]{2}{*}{ Eu- $c_{1}, E A-c_{2}$ (allelic heterogeneity) } & Perfect & 0.892 & 0.870 & 0.832 \\
\hline & Imputed & 0.862 & 0.829 & 0.775 \\
\hline
\end{tabular}

Perfect and imputed data results are given in the upper and lower portion of each cell,

respectively. The sample compositon for each meta-analysis includes European (Eu) and East Asian (EA) whereas causal variants are denoted by $c_{1}$, and $c_{2}$, respectively, for association at $\mathrm{c}_{1}$ and $\mathrm{c}_{2}$

$0.819,0.886$, and 0.865 at respective MAFs of 5,10 , and $20 \%$, whereas the coinciding powers from random-effects meta-analyses were 0.73 , 0.795 , and 0.51 .

In the locus heterogeneity scenario, we found that null cohorts (East Asian, African) had a dramatic impact on the power to detect the causal variant that was associated in only two (European) of the six cohorts (Table 4). In both perfect and imputed data, the average power loss over the regions was $\sim 50 \%$, irrespective of the MAF. The individual region power reductions for perfect data were between 45.7 and $67.9 \%$, with median $60.6 \%$, whereas imputed data had power losses between 52.5 and $75.9 \%$, with median $60.3 \%$. Slightly larger power losses were observed when there was both locus and allelic heterogeneity (different causal variants for European and East Asian, African null cohort), as detailed in Table 4. These results are comparable to previous results ${ }^{10}$ where a fixed-effects analysis of all populations attained a power of $99.7 \%$, whereas the power dropped by 69.3\% when African and East Asian samples were null strata. The power losses from the inclusion of samples with a null association at the variant suggest that locus heterogeneity may have a more profound effect on power compared with allelic heterogeneity. Similar power losses are observed in comparing the single ancestry scenario with any of the heterogeneity settings in the high diversity scenario, as there are negligible differences between the powers of the single ancestry and high diversity ancestral composition in the absence of heterogeneity.

The manner in which the credible set is constructed makes the assumption of a common causal variant that does not hold in the heterogeneity settings, such that a low fine-mapping resolution is expected in these settings. As an illustration, credible sets were constructed when there is moderate diversity and allelic heterogeneity in IGF2BP2, as well as both allelic and locus heterogeneity in the high diversity scenario. Presence of the signal in only one group (Eu) resulted in very large $95 \%$ credible sets, consisting of at least 1500 variants (results not provided). Use of random effects rather than fixed effects reduced the size of the credible sets by $\sim 200$ SNPs, but credible sets were constructed in $<10 \%$ of the replications; few replications had a SNP that passed the genome-wide significance threshold.

\section{DISCUSSION}

There is a clear improvement in fine-mapping resolution when synthesising data across highly diverse samples (European/Asian/ African) in comparison with the analyses of samples from the same ancestry (European). A clear trend of increased fine-mapping
Table 4 Summary measure over the five regions of the power to detect the variant that is causal in the European samples $c_{1}$ (at significance level 0.05 ) in the presence of allelic and locus heterogeneity; positive detection occurs when the $P$-value for the single variant test at $c_{1}$ is below 0.05

\begin{tabular}{|c|c|c|c|c|}
\hline & Data & $M A F$ & $M A F$ & $M A F$ \\
\hline Sample composition and causal variants & type & $5 \%$ & $10 \%$ & $20 \%$ \\
\hline \multirow[t]{2}{*}{ Eu- $_{1}$ (single ancestry) } & Perfect & 1.000 & 1.000 & 1.000 \\
\hline & Imputed & 0.970 & 0.999 & 0.999 \\
\hline \multirow[t]{2}{*}{ Eu/EA/Af- $c_{1}$ (no heterogeneity) } & Perfect & 1.000 & 1.000 & 1.000 \\
\hline & Imputed & 0.998 & 1.000 & 0.999 \\
\hline \multirow{2}{*}{$\begin{array}{l}\text { Eu-c } c_{1}, E A-c_{2}, A f-O \text { (allelic and locus } \\
\text { heterogeneity) }\end{array}$} & Perfect & 0.419 & 0.494 & 0.412 \\
\hline & Imputed & 0.257 & 0.426 & 0.359 \\
\hline \multirow[t]{2}{*}{ Eu-C $C_{1}, E A / A f-0$ (locus heterogeneity) } & Perfect & 0.413 & 0.539 & 0.432 \\
\hline & Imputed & 0.320 & 0.495 & 0.399 \\
\hline
\end{tabular}

Perfect and imputed data results are given in the upper and lower portion of each cell, respectively. The sample compositon for each meta-analysis includes European (Eu), East Asian $(E A)$, and African (Af), whereas causal variants are denoted by $0, c_{1}$, and $c_{2}$, respectively, for null data, association at $\mathrm{c}_{1}$, and association at $\mathrm{c}_{2}$.

resolution is not apparent in comparisons between common ancestry meta-analyses and those consisting of samples of European and Asian ancestry. This may be because of individuals of European and Asian ancestry being more closely related with each other than with those of African ancestry, as had been illustrated in previous relatedness analyses. ${ }^{9}$ These results indicate that the meta-analysis of ancestrally diverse samples results in an improvement in fine-mapping resolution, provided that the composition includes those of African ancestry. For further investigation of this improvement, we examined African-only meta-analyses that displayed a high fine-mapping resolution, suggesting that increasing the sample size of African ancestry samples is more likely to improve fine-mapping resolution than increasing sample diversity. It would also be of interest to investigate the inclusion of Hispanic cohorts, given the current efforts in establishing large Hispanic cohorts, the admixing history, and ethnic diversity within such cohorts. Given the fine-mapping improvements observed from the inclusion of African ancestry samples, and that the LD levels in Hispanic samples tend to fall between those of Africans and Europeans/East Asian, inclusion of Hispanic samples is expected to contribute to refinement in fine-mapping over that of European and East Asian samples.

Methods for trans-ethnic meta-analyses have been compared in extensive simulation studies, ${ }^{10}$ yet the impact of ancestral composition in study design has not previously been investigated. Although the conducted simulation studies are limited by taking place under an ideal scenario in which imputation reference panels consist of populations that are exact matches for the study samples, they act as a gauge for the performance of the various ancestral composition study designs. This study highlights the benefits of trans-ethnic metaanalysis for detecting novel complex trait loci and fine-mapping causal variants, when there is a shared causal variant. It also considers the effect on power from the inclusion of cohorts where the variant is null that may occur if it was discovered in African populations and did not segregate outside of Africa, or vice versa. In this case, the fixed-effects (and random-effects) meta-analysis is exposed to a dramatic power loss when the sample size is fixed over the different ancestral diversity scenarios. Therefore, when there is a restriction on sample size (eg, cost), careful consideration is needed in designing the ancestral 
composition for the meta-analysis, depending on the assumption of shared causal variants or locus heterogeneity.

Meta-analyses are often performed with an intention of maximising the number of available cohorts, with or without African ancestry cohorts. This is often owing to the restricted opportunities to include African cohorts, either because of GWAS data not existing or not readily available for sharing and for meta-analysis. Our simulation results indicate the importance of including African ancestry samples in fine-mapping studies, and that high gains in resolution are possible with their inclusion. Considering the high resolution in the Africanonly analyses, it may be beneficial to include a few small African cohorts (eg, size 1000 each) rather than an additional couple of large European cohorts (eg, size $>10000$ ). The possibility of deriving benefits that have a global impact may therefore be more likely by developing the capacity for genomic sciences in Africa, such that an increased number of African-focussed GWASs may be performed. This includes enabling and empowering more African researchers to carry out such studies in their populations.

\section{CONFLICT OF INTEREST}

The authors declare no conflict of interest.

\section{ACKNOWLEDGEMENTS}

We thank three anonymous reviewers and the section editor for their helpful and insightful comments that have improved the clarity of the manuscript. This research is supported by the Wellcome Trust (WT098051) and the National Institutes of Health award for the T2D-GENES project (4 U01 DK085545-05). JLA is funded by the Medical Research Council Methodology Research Fellowship (MR/K021486/1). APM is a Wellcome Trust Senior Research Fellow in Basic Biomedical Science (Grant Number WT098017).

1 Welter D, MacArthur J, Morales J, Burdett T, Hall P, Junkins H: The NHGRI GWAS catalog, a curated resource of SNP-trait associations. Nucleic Acids Res 2014; 42: D1001-D1006.

2 Zaitlen N, Paşaniuc B, Gur T, Ziv E, Halperin E: Leveraging genetic variability across populations for the identification of causal variants. Am J Hum Genet 2010; 86: 23-33.
3 Cooper RS, Tayo B, Zhu X: Genome-wide association studies: implications for multiethnic samples. Hum Mol Genet 2008; 17: R151-R155.

4 Gurdasani D, Carstensen T, Tekola-Ayele F et al: The African Genome Variation Project shapes medical genetics in Africa. Nature 2015; 517: 327-332.

5 DIAbetes Genetics Replication And Meta-analysis (DIAGRAM) Consortium, Asian Genetic Epidemiology Network Type 2 Diabetes (AGEN-T2D) Consortium, South Asian Type 2 Diabetes (SAT2D) Consortiumet al: Genome-wide trans-ancestry meta-analysis provides insight into the genetic architecture of type 2 diabetes susceptibility. Nat Genet 2014; 46: 234-244.

6 Liu CT, Buchkovich ML, Winkler TW et al: Multi-ethnic fine-mapping of 14 central adiposity loci. Hum Mol Genet 2014; 23: 4738-4744.

7 Wu Y, Waite LL, Jackson AU et al: Trans-ethnic fine-mapping of lipid loci identifies population-specific signals and allelic heterogeneity that increases the trait variance explained. PLoS Genet 2013; 9: e1003379.

8 Mägi R, Morris AP: GWAMA: software for genome-wide association meta-analysis. BMC Bioinformatics 2010; 11: 288.

9 Morris AP: Trans-ethnic meta-analysis of genome-wide association studies. Genet Epidemiol 2011; 35: 809-822.

10 Wang $\mathrm{X}$, Chua HX, Chen $\mathrm{P}$ et al: Comparing methods for performing trans-ethnic meta-analysis of genome-wide association studies. Hum Mol Genet 2013; 22: 2303-2311.

11 Waters KM, Stram DO, Hassanein MT et al: Consistent association of type 2 diabetes risk variants found in Europeans in diverse racial and ethnic groups. PLoS Genet 2010; 6: e1001078.

12 Su Z, Marchini J, Donnelly P: HAPGEN2: simulation of multiple disease SNPs. Bioinformatics 2011; 27: 2304-2305.

13 The 1000 Genomes Project Consortium: A map of human genome variation from population-scale sequencing. Nature 2010; 467: 1061-1073.

14 Howie BN, Donnelly P, Marchini J: A flexible and accurate genotype imputation method for the next generation of genome-wide association studies. PLoS Genet 2009; 5: e1000529.

15 Marchini J, Howie B, Myers S, McVean G, Donnelly P.: A new multipoint method for genome-wide association studies via imputation of genotypes. Nat Genet 2007; 39: 906-913.

16 Wakefield J: Bayes factors for genome-wide association studies: comparison with p-values. Genet Epidemiol 2009; 33: 79-86.

17 Maller JB, McVean G, Byrnes J et al: Bayesian refinement if association signals for 14 loci in 3 common diseases. Nat Genet 2012; 44: 1294-1301.

This work is licensed under a Creative Commons Attribution 4.0 International License. The images or other third party material in this article are included in the article's Creative Commons license, unless indicated otherwise in the credit line; if the material is not included under the Creative Commons license, users will need to obtain permission from the license holder to reproduce the material. To view a copy of this license, visit http:// creativecommons.org/licenses/by/4.0/

Supplementary Information accompanies this paper on European Journal of Human Genetics website (http://www.nature.com/ejhg) 